\title{
TRANSVERSUS ABDOMINIS PLANE (TAP) BLOCK REDUCES POSTOPERATIVE MORHPINE REQUIRMENT AND POSTOPERATIVE SEDATION SCORES IN PATIENTS UNDERGOING LOWER ABDOMINAL OPERATIONS: A PROSPECTIVE STUDY.
}

Melchisedec, Anil Luther, Anish. G. Varghese, Valsa Verghese

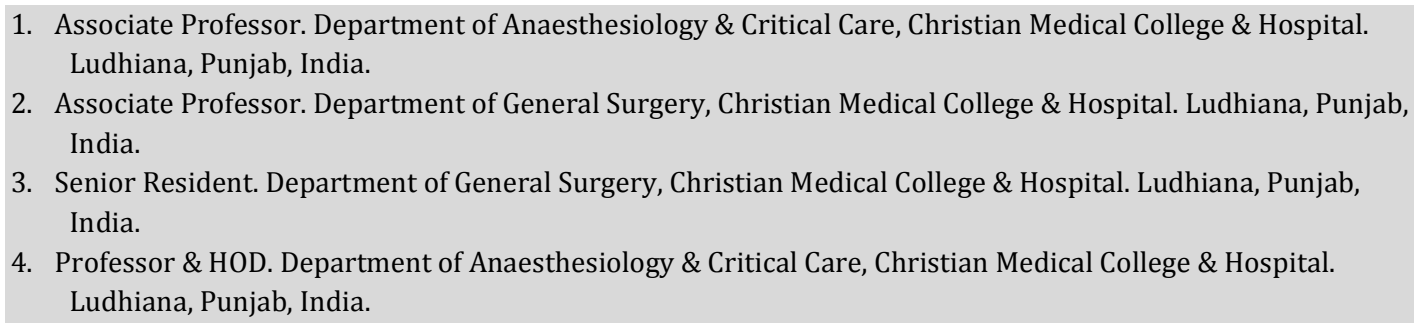

\section{CORRESPONDING AUTHOR:}

Dr. Melchisedec,

Associate Professor,

Department of Anaesthesiology and Critical Care,

Christian Medical College and Hospital,

Ludhiana, Punjab, India. 141008.

E-mail: drmelchisedec@yahoo.com

ABSTRACT: BACKGROUND: Transversus abdominis plane (TAP) block is a regional anaesthetic technique which has recently gained popularity. It is being used for postoperative analgesia for upper and lower abdominal surgeries. Local anaesthetic is deposited in the neuro-fascial plane between the internal oblique and the transversus abdominis muscle. We carried on this study to assess the postoperative pain and sedation scores in patients undergoing lower abdominal surgeries in a randomized controlled, double blind study. METHODS: 40 patients scheduled to undergo inguinal hernia repair or appendicectomy were randomly selected. All the patients received a general anaesthetic for the proposed surgery. Patients were divided into two groups, a Tab block group in which the patients were given a Tap block after the induction of general anaesthetic and a control group in which the patients did not receive the Tap block. Patients in both the groups received Inj acetaminophen 1gm every 8 hourly and Inj morphine $0.05 \mathrm{mg} / \mathrm{kg}$ intermittently every time the patient complaint of pain postoperatively. Patients in both the groups were assessed for postoperative morphine requirements and sedation scores at 2,6,12 and 24 hours. RESULTS: There was a significant difference in the verbal analogue scores for pain as well as the visual analogue scores for sedation in both the groups. Postoperative morphine requirement (24hours) was significantly reduced in the patients who did not receive the Tap block as compared to the patients who receive the block (17.5mg Vs $7 \mathrm{mg}$ ). Postoperative sedation scores were also significantly less in the Tap block group as compared to the control group. CONCLUSIONS: Tap block provides a highly effective analgesia in patients undergoing lower abdominal surgery. It reduces the morphine requirement and sedation scores in the 24 hours postoperative period.

INTRODUCTION: The transversus abdominis plane (TAP) block is a regional anesthesia technique that provides analgesia to the parietal peritoneum as well as the skin and muscles of the anterior abdominal wall [1]. A substantial component of the pain experienced by patients 
after abdominal surgery is derived from the abdominal wall incision. The skin, muscles, and parietal peritoneum of the anterior abdominal wall are innervated by the lower six thoracic nerves and the first lumbar nerve. The anterior primary rami of these nerves leave their respective intervertebral foramina and course over the vertebral transverse process. They then pierce the musculature of the lateral abdominal wall to course through a neuro-fascial plane between the internal oblique and transversus abdominis muscles [2]. The sensory nerves branch first in the mid-axillary line sending out a lateral cutaneous branch, and continue within the plane to perforate anteriorly supplying the skin as far as the midline.

The transversus abdominis plane thus provides a space into which local anesthetic can be deposited to achieve myocutaneous sensory blockade. Deposition of the local anesthetic dorsal to the mid-axillary line also blocks the lateral cutaneous afferents, thus facilitating blockade of the entire anterior abdominal wall. The lumbar triangle of Petit offers an easily identifiable, fixed and palpable landmark, and is located dorsal to the mid-axillary line [3].The transversus abdominis neuro-fascial plane can easily be accessed via this triangle using the double loss of resistance technique and local anesthetic deposited into this plane [4].

MATERIALS AND METHOD: The aim of the study was to see the efficacy of TAP block in reducing postoperative morphine requirements and postoperative sedation scores after lower abdominal surgery. The study included 40 patients ASA ll-lll, scheduled for appendicectomy or inguinal hernia repair. A written informed consent was taken from each patient. Patients with history of allergy to local anaesthetics, infection at the site of injection, inability to co-operate with the treatment were not included in the study.

Patients were randomized, to undergo a unilateral TAP block $(n=20)$ or to receive standard care(control group, $n=20$ ). The patients, anesthesiologists, and the staff providing postoperative care were blinded to group assignment. A unilateral TAP block was given on the side of the pathology. All patients received a standardized general anesthetic. Standard monitoring, including electrocardiogram, arterial blood pressure, arterial oxygen saturation, and end-tidal carbon dioxide monitoring were used throughout the surgery. Anaesthesia was induced with inj sodium thiopentone $(3-5 \mathrm{mg} / \mathrm{kg})$, Inj fentanyl $(1 \mathrm{ug} / \mathrm{kg})$ and Inj succinylcholine 1-2 mg kg. Atracurium was used for muscle relaxation. Anaesthesia was maintained with oxygen, nitrous oxide and isoflurane. All patients randomized to undergo TAP block had the block performed using the loss of resistance technique after the induction of anesthesia. The iliac crest was palpated from anterior to posterior until the latissimus dorsi muscle could be felt. The triangle of Petit was then located just anterior to the latissimus dorsi muscle. Using a 20G Tuohy needle, the skin was pierced just cephalad to the iliac crest over the triangle of Petit. The needle was then advanced at right angles to the skin, in a coronal plane, until resistance was encountered. This resistance indicated that the needle tip was at the external oblique muscle. Gentle advancement of the needle resulted in a "pop" sensation as the needle entered the plane between the external and internal oblique fascial layers. Further gentle advancement of the needle resulted in a second pop, which indicated entry into the transversus abdominis fascial plane. After careful aspiration to exclude vascular puncture, $20 \mathrm{~mL}$ of $0.5 \%$ bupivacaine solution was then injected through the needle.

After completion of the surgical procedure, and emergence from anesthesia, patients were transferred to the recovery ward. Patients in both the groups were started on a standard postoperative analgesic regimen after the first demand for pain relief, consisting of iv acetaminophen $1 \mathrm{~g}$ every 8 hourly and Inj morphine $0.05 \mathrm{mg} / \mathrm{kg}$ intermittently when required . The presence and severity of pain and sedation was assessed systematically by an investigator 
blinded to the group allocation. These assessments were performed in the recovery room at 2,6,12 and 24 hours postoperatively. All patients were asked to give scores for their pain. Pain severity was measured using both a visual analog scale ( $0=$ no pain, $10=$ worst imaginable) and a categorical pain scoring system (none $=0$; mild $=1$; moderate $=2$; severe $=3$ ). Sedation scores were assigned by the blinded assessor, using a sedation scale (awake and alert $=0$; quietly awake $=1$; asleep but easily aroused $=2$; deep sleep $=3$ ).

RESULTS: Forty patients were entered into the study. Of the 40 patients, 20 were randomized to undergo TAP blockade and 20 patients received standard care (control group).Groups were comparable in terms of age, weight, height, ASA status and duration of surgery. Patients undergoing inguinal hernia repair and appendicectomy were taken for the study so that both the groups were comparable. In all the patients included in the study the lumbar triangle of petit was easily felt and the transversus abdominis plane was easily located. It was seen that there was a significant difference in the time for first morphine between the control group (1hr) and the tap block group(8hrs).There was a significant difference in the postoperative verbal analogue pain scores among the two groups at all the times. The visual analogue scores for sedation were also significantly lower in the patients who received the TAP block. Postoperative morphine requirement between the two groups was significantly different among the two groups, $17.5 \mathrm{mg}$ in the control group and $7 \mathrm{mg}$ in the tap block group ( $\mathrm{p}$ value $<0.05$ ).

DISCUSSION: A promising approach to the provision of postoperative analgesia after abdominal incision is to block the sensory nerve supply to the anterior abdominal wall.[5] However, the clinical utility of current approaches to the blockade of these nerve afferents, such as abdominal field blocks, is limited, and the degree of block achieved can be unpredictable. A major reason for the relative lack of efficacy of these blocks is the lack of clearly defined anatomic landmarks, leading to uncertainty regarding the exact needle positioning, and the lack of a clear indication that the local anesthetic is being deposited in the correct anatomical plane.

The analgesic efficacy of the TAP block has been demonstrated in prospective randomized trials compared with placebo, in different surgical procedures such as abdominal surgery[6], hysterectomy[7], retropubic prostatectomy[8], Caesarean section[9], and laparoscopic cholecystectomy.[10] All the studies have reported superiority of the TAP block in terms of reduction in visual analogue scale scores and morphine consumption.

Pain after surgery for acute appendicitis has two sources, namely the somatosensory pain originating from the surgical wound on the anterior abdominal wall and the visceroperitonitic pain due to the inflammation and infection the appendix [11] TAP block ameliorates the pain from the surgical wound.[12]

A single injection can achieve sensory block over a wide area of the abdominal wall. The block has been shown to be useful in upper abdominal surgery, but the upper extent of the block and its use in upper abdominal surgery are controversial.

TAP block is particularly useful for cases when an epidural is contraindicated or refused. The block can be performed unilaterally eg. for Appendicectomy or bilaterally when the incision crosses the midline eg. in Pfannenstiel incision. A single injection can be used, or a catheter inserted for several days for analgesic benefit. TAP block also has a role as rescue analgesia on awake postoperative patients who did not receive blocks prior to abdominal surgery.

Regional anaesthesia in general has a very low rate of serious complications. The risk in regional anaesthesia varies with the type and location of the block. General risks of regional blockade include: needle trauma, intraneural injection, neural ischemia, inadvertent 
intravascular injection, local anaesthetic toxicity, infection and poor/failed block. The general risks for regional blockade are applicable to the TAP block, however the site of injection for the TAP block is relatively low risk. The landmark technique relies on the 'pop' sensation which some clinicians believe is an imprecise sign. The identification of the landmarks is more challenging in the obese hence the risk of peritoneal perforation is probably higher. In our study two obese patients in whom the landmarks for the tap block were not clear, were not included in the study as our objective was to assess the postoperative morphine requirement and sedation scores. If anatomy is abnormal, such as hepatomegaly, there is risk of damage from the needle puncture. [13]

A potential drawback of tap block includes that a bilateral block is required in most surgical procedures, and, in addition, the duration of the block may be limited to a few hours and could be too short to guarantee a pain free postoperative course. To avoid this disadvantage, a catheter could be placed for continuous local anaesthetic infusion but only on one side.

A significant advantage of this block can be its use as a sole regional anaesthetic in patients who are high risk for general anaesthesia or in those in whom an epidural or spinal is contraindicated.

In this study postoperative morphine requirement was significantly reduced. Although intermittent bolus doses of $0.05 \mathrm{mg} / \mathrm{kg}$ were given whenever patient required an analgesic, a patient controlled analgesia with the use of a pain pump could have been a more specific approach in assessing the postoperative morphine requirement.

Ultrasound techniques are likely to improve the safety of this block as the needle passage and injection can be followed in real time [14] however this has not been scientifically tested to date. Some authors argue that peritoneal perforation with a small gauge sterile needle is not likely to be significant.

Ultrasound guidance for performance of this block has become the method of choice and it was not done in our study because of its non-availability. We can look forward to conduct more research on this topic with the help of an ultrasound to go for wider range of possible techniques, particularly for targeting the upper abdominal wall. The ease with which this block can be performed, an excellent safety profile to date and outstanding clinical utility, will no doubt lead to increase in popularity and use of the transversus abdominis plane block.

\section{Baseline patient characteristics:}

Table1:Baseline patient characteristics:

\begin{tabular}{|l|c|c|}
\hline Group & Control (n=20) & Tap Block (n=20) \\
\hline Age (yrs) & $33 \pm 9$ & $30 \pm 6$ \\
\hline Sex ratio (M: F) & $14: 6$ & $12: 8$ \\
\hline Weight (kg) & $68 \pm 9$ & $66 \pm 8$ \\
\hline Height (m) & $1.50 \pm 06$ & $1.53 \pm 04$ \\
\hline Duration of surgery (mins) & $68 \pm 8$ & $65 \pm 10$ \\
\hline Surgical procedure: & 20 & 20 \\
\hline Inguinal hernia repair: & 12 & 11 \\
\hline Appendicectomy: & 8 & 9 \\
\hline
\end{tabular}


Table2: Postoperative Pain and Sedation Scores:

\begin{tabular}{|l|c|c|}
\hline Group & Control (n=20) & Tap Block(n=20) \\
\hline VAS pain & 1 & 0 \\
\hline $2 \mathrm{hr}$ & 1 & 0 \\
\hline $6 \mathrm{hr}$ & 3 & 0 \\
\hline $12 \mathrm{hr}$ & 2 & 1 \\
\hline $24 \mathrm{hr}$ & & 1 \\
\hline VAS Sedation & 1 & 0 \\
\hline $2 \mathrm{hr}$ & 2 & 1 \\
\hline $6 \mathrm{hr}$ & 3 & 1 \\
\hline $12 \mathrm{hr}$ & 1 & $8^{*}$ \\
\hline $24 \mathrm{hr}$ & 1 & $7^{*}$ \\
\hline Mean Time for 1st request for morphine(Hrs) & 17.5 & \\
\hline Mean 24hr morphine requirement(mg) & & \\
\hline
\end{tabular}

$\mathrm{p}$ value $<0.05$

\section{REFERENCES:}

1. Rafi AN. Abdominal field block: a new approach via the lumbar triangle, Anaesthesia 2001. 56; 10: 1024-1026.

2. Rozen WM, Tran TMN, Ashton MW, Barrington MJ, Ivanusic JJ, Taylor GI. Refining the course of the thoracolumbar nerves. A new understanding of the innervations of the anterior abdominal wall. Clin Anat 2008; 21: 325-33

3. Netter FH. Abdomen posterolateral abdominal wall. In: Netter FH, ed. Atlas of human anatomy summit. New Jersey, USA: The Ciba-Geigy Corporation, 1989:230-40.

4. McDonnell JG, O'Donnell BD, Farrell T, et al. Transversus abdominis plane block: a cadaveric and radiological evaluation. Reg Anesth Pain Med 2007; 32: 399-404.

5. Niraj G, Kelkar A, Fox A. Application of the transversus abdominis plane (TAP) block in the intensive care unit. Anaesth Intensive Care 2009; 37

6. McDonnell JG, O'Donnell BD, Curley GCJ, et al. The analgesic efficacy of transversus abdominis block after abdominal surgery. Anesth Analg 2007; 104: 193-7

7. Carney J, McDonnell JG, Ochana A, Bhinder R, Laffey JG. The transversus abdominis plane block provides effective postoperative analgesia in patients undergoing total abdominal hysterectomy. Anesth Analg 2008; 107: 2056-60

8. O'Donnell BD, McDonnell JG, McShane AJ. The transversus abdominis plane (TAP) block in open retropubic prostatectomy. Reg Anesth Pain Med 2006; 31: 91

9. McDonnell JG, Curley GCJ, Carney J, et al. The analgesic efficacy of transversus abdominis block after cesarean delivery. Anesth Analg 2008; 106: 186-91

10. El-Dawlatly AA, Turkistani A, Kettner SC, et al. Ultrasound guided transverses abdominis plane block: description of a new technique and comparison with conventional systemic analgesia during laparoscopic cholecystectomy. Br J Anaesth 2009; 102: 763-7

11. Aida S, Baba H, Yamakura T, Taga K, Fukuda S, Shimoji K. The effectiveness of preemptive analgesia varies according to the type of surgery: a randomized, double-blind study. Anesth Analg 1999; 89: 711-6

12. Niraj G, Searle A, Mathews M, et al. Analgesic efficacy of ultrasound-guided transversus abdominis plane block in patients undergoing open appendicectomy. Br J Anaesth 2009; 103: $601-5$ 
13. Farooq M, Carey M. A case of liver trauma with a blunt regional anesthesia needle while performing transversus abdominis plane block. Reg Anesth Pain Med 2008; 33: 27

14. Shibata Y, Sato Y, Fujiwara Y, Komatsu T. Transversus abdominis plane block. Anesth Analg 2007;105:883

Figure 1. Line drawing of a transverse section through the abdominal wall at the level of the lumbar triangle of Petit.

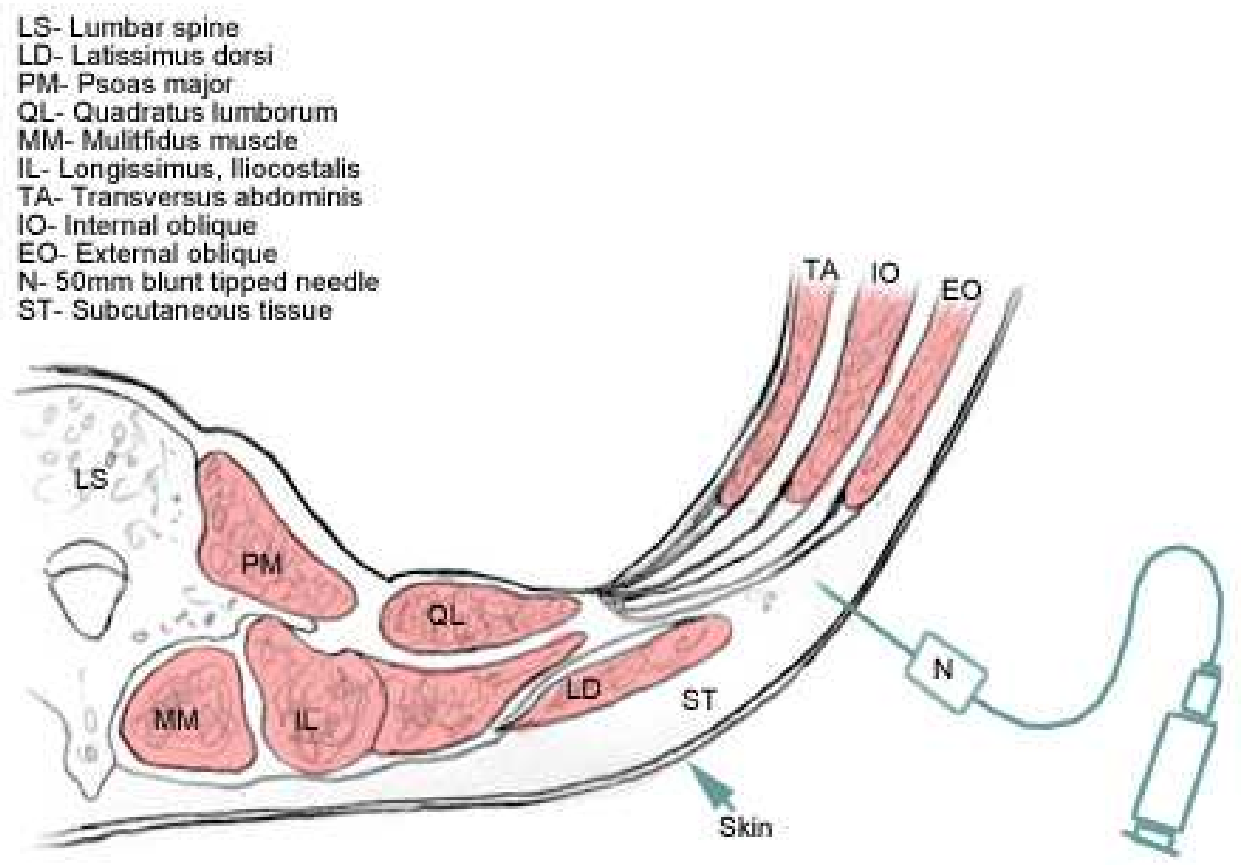




\section{ORIGINAL ARTICLE}

Figure 2.Surface anatomy labeled for landmark insertion of TAP block in an adult male in the supine position.

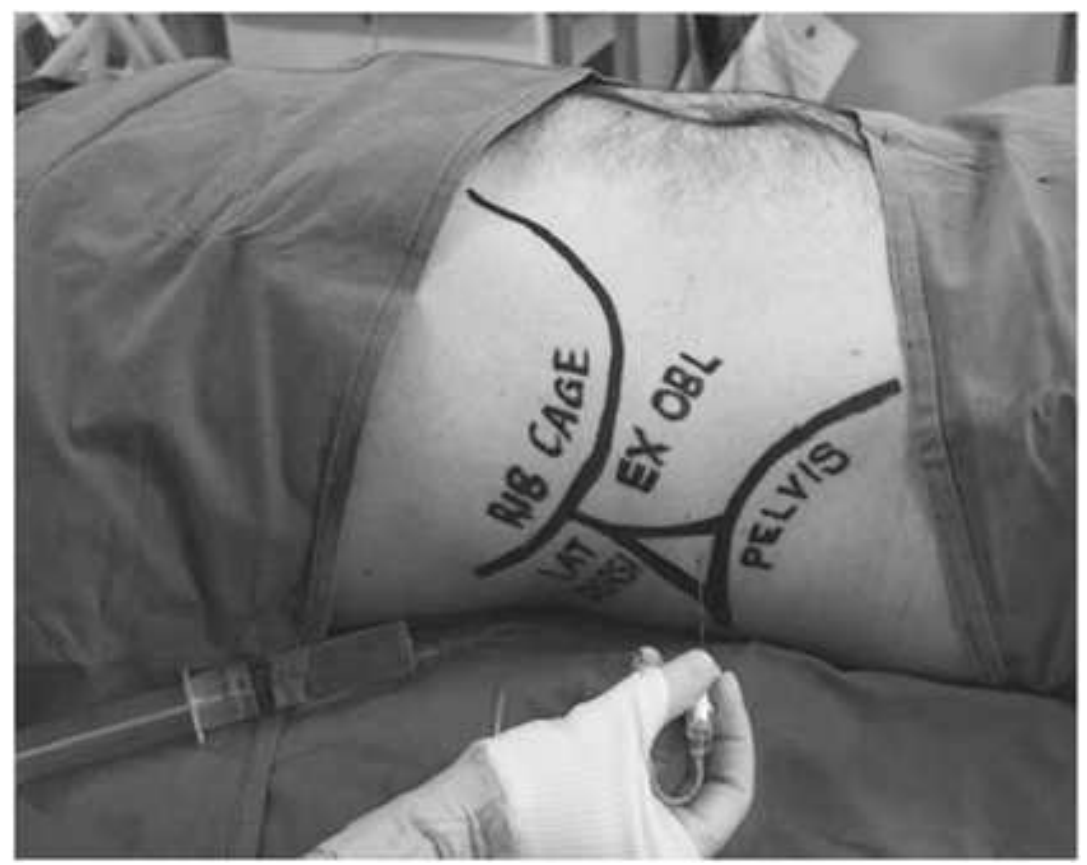

